\title{
Virtual Private Network Design Under Traffic Uncertainty
}

\author{
A. Altın ${ }^{\mathrm{a}, 1}$, E. Amaldi ${ }^{\mathrm{b}, 2}$, P. Belotti ${ }^{\mathrm{b}, 3}$, M. Ç. Pınar ${ }^{\mathrm{a}, 4}$ \\ a Bilkent University, Department of Industrial Engineering, 06800, Bilkent, Ankara, Turkey \\ ${ }^{\mathrm{b}}$ Politecnico di Milano, Dipertimento di Elettronica e Informazione, Piazza Leonardo da Vinci, \\ 32, 20133 Milano, Italy
}

\begin{abstract}
We propose different formulations as well as efficient solution approaches for the VPN design problem under traffic uncertainty with symmetric bandwidths.
\end{abstract}

Keywords: VPN design, traffic uncertainty.

\section{Introduction}

A Virtual Private Network (VPN) service is similar to a private network service since it enables a group of nodes over a large underlying network to communicate with each other using an already available physical network like the Internet.

In this paper we deal with the polyhedral model of Ben-Ameur and Kerivin (2002), which allows the traffic vector to belong to a polytope defined by some customer specific constraints. We offer three different formulations for the VPN design problem where the solution is allowed to be an arbitrary

$\overline{1}$ Email: aysegula@bilkent.edu.tr

2 Email: amaldi@elet.polimi.it

3 Email: belotti@elet.polimi.it

4 Email: mustafap@bilkent.edu.tr 
subgraph. We propose a Column Generation and a Cutting Plane Algorithm to solve these formulations efficiently.

We assume that we are given an undirected network $G=(V, E)$ and a set of VPN sites $W \subseteq V$. Each edge $\{i, j\} \in E$ is assigned a unit capacity reservation $\operatorname{cost} c_{i j}>0$. The aggregate traffic inflow and outflow bandwidths for each terminal $s \in W$ is symmetric, i.e. $b_{s}^{+}=b_{s}^{-}=b_{s} \forall s \in W$. A traffic demand vector is $\vec{d}=\left(d_{s t}\right)_{s \in W, t \in W \backslash\{s\}}$. The set of demand pairs is $Q=\{(s, t): s, t \in W, s \neq t\}$ and we are given a matrix $A \in \Re^{h *|Q|}$ where $h$ is the number of constraints defining the traffic polytope $D=\left\{\vec{d}: A \vec{d} \leq \vec{a}, \vec{a} \in \Re^{h}\right\}$. Each terminal $s \in W$ is required to route its traffic to site $t \in W \backslash\{s\}$ unsplittably on a single path $P_{s t}$ and the final solution, i.e., $P=\bigcup_{(s, t) \in Q} P_{s t}$, is allowed to be an arbitrary subgraph of $G=(V, E)$. The problem is to find a least cost capacity installation so as to satisfy all possible traffic demands known to lie in the polytopic set $D$.

\section{The Polyhedral Flow Formulation}

In this section we present the following mixed IP formulation of the problem using the binary flow variable $y_{i j}^{s t}$, which is 1 if the directed arc $(i, j)$ is contained in the path going from terminal $s$ to terminal $t$.

$$
\begin{aligned}
& \min \sum_{\{i, j\} \in E} c_{i j} x_{i j} \\
& \text { s.t. } \sum_{j:\{i, j\} \in E}\left(y_{i j}^{s t}-y_{j i}^{s t}\right)=\left\{\begin{array}{r}
1 i=s \\
-1 i=t \quad \forall i \in V,(s, t) \in Q \\
0 \text { o.w. }
\end{array}\right. \\
& \sum_{(s, t) \in Q} d_{s t}\left(y_{i j}^{s t}+y_{j i}^{s t}\right) \leq x_{i j} \quad \forall\{i, j\} \in E \\
& A \vec{d} \leq \vec{a} \\
& y_{i j}^{s t} \in\{0,1\} \\
& x_{i j} \geq 0 \\
& \begin{array}{l}
\forall\{i, j\} \in E,(s, t) \in Q \\
\forall\{i, j\} \in E
\end{array}
\end{aligned}
$$

where the constraints (2) are the flow constraints. Constraint set (3) defines the amount of capacity reserved on the edge $\{i, j\}$ considering all feasible traffic scenarios defined by (4). Note that the constraints (3) are nonlinear. Our contribution at this point is to linearize these constraints so as to obtain the following compact mixed-integer formulation. 
(5)

$$
\min \sum_{\{i, j\} \in E} c_{i j} x_{i j}
$$

(6) s.t. $\sum_{j:\{i, j\} \in E}\left(y_{i j}^{s t}-y_{j i}^{s t}\right)=\left\{\begin{array}{c}1 i=s \\ -1 i=t \quad \forall i \in V,(s, t) \in Q \\ 0 \text { o.w. }\end{array}\right.$

$$
\begin{array}{cl}
{\overrightarrow{w^{i j}}}^{T} \vec{a} \leq x_{i j} & \forall\{i, j\} \in E \\
{\overrightarrow{w^{i j}}}^{T} A^{T} \geq \overrightarrow{y_{i j}}+\overrightarrow{y_{j i}} & \forall\{i, j\} \in E \\
x_{i j} \geq 0, \overrightarrow{w^{i j}} \geq 0 & \forall\{i, j\} \in E \\
y_{i j}^{s t} \in\{0,1\} & \forall\{i, j\} \in E,(s, t) \in Q
\end{array}
$$

where $\overrightarrow{w^{i j}}$ is the vector of dual variables.

\section{The Polyhedral Path Formulation}

In this case we have the binary variable $z_{p}$, which is 1 if traffic is routed through path $p$ in the optimal solution. Then our path formulation is

(12) s.t.

$$
\begin{array}{ll}
\min \sum_{\{i, j\} \in E} c_{i j} x_{i j} & \\
\sum_{p \in P_{s t}} z_{p} \geq 1 & \forall(s, t) \in Q
\end{array}
$$

$$
\sum_{(s, t) \in Q} d_{s t} \sum_{p \in P_{s t} \cap P_{\{i, j\}}} z_{p} \leq x_{i j} \forall\{i, j\} \in E
$$

$$
\begin{array}{cl}
A \vec{d} \leq \vec{a} & \\
z_{p} \in\{0,1\} & \forall(s, t) \in Q, p \in P_{s t} \\
x_{i j} \geq 0 & \forall\{i, j\} \in E
\end{array}
$$

where (12) ensures that the demand pair $(s, t) \in Q$ communicates and (13) defines the capacity reservation on edge $\{i, j\}$ considering all possible traffic scenarios defined as in (14). Note that type (13) constraints are nonlinear and can be linearized judiciously to obtain

(18) s.t.

$$
\begin{gathered}
\min \sum_{\{i, j\} \in E} c_{i j} \sum_{k=1}^{h} a_{k} w_{k}^{i j} \\
\sum_{p \in P_{s t}} z_{p} \geq 1 \quad \forall(s, t) \in Q
\end{gathered}
$$




$$
\begin{array}{cc}
\sum_{k=1}^{h} a_{k, s t} w_{k}^{i j} \geq \sum_{p \in P_{s t} \cap P_{\{i, j\}}} z_{p} & \forall(s, t) \in Q,\{i, j\} \in E \\
z_{p} \in\{0,1\} & \forall(s, t) \in Q, p \in P_{s t} \\
\overrightarrow{w^{i j}} \geq 0 & \forall\{i, j\} \in E
\end{array}
$$

We propose to use a column generation algorithm to solve the path formulation,which can be summarized as follows:

- Step 0 Let the inital set of paths include the pairwise shortest paths.

- Step 1 Solve the path formulation with the current set of paths.

- Step 2 For each $(s, t) \in Q$ if you find a path $p$ such that $\sigma_{s t}-\sum_{\{i, j\} \in p} \pi_{i j}^{s t}>0$, the add it to te current set of paths.

- Step 3 Go to step 1 if new paths are added in Step 2. Otherwise stop!

\section{The Polyhedral Cut Formulation}

In this case we require connectivity over all cuts. Then the polyhedral cut formulation is as given below.

$$
\begin{aligned}
& \text { (22) } \quad \min \sum_{\{i, j\} \in E} c_{i j} \overrightarrow{a^{T}} \overrightarrow{w^{i j}} \\
& \text { (23) s.t. } \sum_{\{i, j\} \in \delta(W)} \overrightarrow{w^{i j}} A_{s t} \geq 1 \forall(s, t) \in Q \\
& \text { (24) } \overrightarrow{w^{i j}} \geq 0 \quad \forall\{i, j\} \in E
\end{aligned}
$$

where $A_{s t}$ denotes the column of $A$ corresponding to the demand pair $(s, t) \in$ $Q$. We propose to use a cutting plane algorithm to solve the above problem, which can be summarized as follows.

- Step 0 Set the current cut to empty set.

- Step 1 Solve the above problem with the current cut set.

- Step 2 For each demand pair $(s, t) \in Q$ use the max flow-min cut theorem to determine the violated cut inequalities of type (23). If you find such an edge, then add that edge to the current cut.

- Step3 If the cut set is updated, then go to Step 1, otherwise stop!

\section{References}

Ben-Ameur, W., Kerivin, H., 2002. Routing of Uncertain Demands. submitted. 